\title{
Research Article \\ Effects of Reentry Plasma Sheath on Mutual-Coupling Property of Array Antenna
}

\author{
B. W. Bai, ${ }^{1}$ X. P. Li, ${ }^{1}$ Y. M. Liu, ${ }^{1}$ and J. $X u^{2}$ \\ ${ }^{1}$ School of Aerospace Science and Technology, Xidian University, Xian, Shaanxi 710071, China \\ ${ }^{2}$ School of Electronic Engineering, Xidian University, Xian, Shaanxi 710071, China \\ Correspondence should be addressed to B. W. Bai; bwbai@stu.xidian.edu.cn
}

Received 31 December 2014; Revised 20 March 2015; Accepted 23 March 2015

Academic Editor: Ding-Bing Lin

Copyright ( 2015 B. W. Bai et al. This is an open access article distributed under the Creative Commons Attribution License, which permits unrestricted use, distribution, and reproduction in any medium, provided the original work is properly cited.

\begin{abstract}
A plasma sheath enveloping a reentry vehicle would cause the failure of on-board antennas, which is an important effect that contributes to the "blackout" problem. The method of replacing the on-board single antenna with the array antennas and using beamforming technology has been proposed to mitigate "blackout" problem by many other researchers. Because the plasma sheath is a reflective medium, plasma will alter the mutual coupling between array elements and degrade the beamforming performance of array antenna. In this paper, the effects of the plasma sheath on the mutual coupling properties between adjacent array elements are studied utilizing the algorithm of finite integration technique. Results show that mutual coupling coefficients of array elements are deteriorating more seriously with the decrease of collision frequency. Moreover, when electron density and collision frequency are both large, plasma sheath improves the mutual coupling property of array elements; this conclusion suggests that replacing the on-board single antenna with the array antennas and using beamforming technology can be adopted to mitigate the blackout problem in this condition.
\end{abstract}

\section{Introduction}

A spacecraft reentering the Earth's atmosphere at a hypersonic speed is enveloped by plasma fluid because of the shock wave heating of ambient gas and ablating of heat shield, causing air molecules and heat shield material dissociated and ionized [1]. Plasma fluid is called reentry plasma sheath, and it contains so many free electrons and ions that it attenuates radio frequency signals greatly through the reflection and absorption effects. The primary problem [2] associated with the reentry plasma sheath is the "communications blackout" phenomenon during which performance of on-board electromagnetic systems can be severely degraded or even completely invalid. The problem is of great importance, because the phase of losing tracking, telemetry, and command (TT\&C) data and GPS navigation signals up to several minutes is often the most crucial part of the vehicle's flight. Thus, the problem has been studied since the Gemini and
Apollo space programs and attracts more attentions with the increasing space activity.

One of many significant effects that contribute to the "blackout" problem is the failure of on-board antenna which is affected greatly by the reentry plasma sheath [3]. Performance degradations and failures of on-board antennas covered by the reentry plasma sheath have been studied by many authors for several decades. Swift et al. [4-6] analysed characteristics of S-band telemetry slot and waveguide antennas radiating into the nonuniform reentry plasma sheath. Galejs [7-9] analysed and measured admittance deviations of plasma-covered slot antenna using the variational method. Based on the impedance sheet notion, Fante [10] described a simple technique for the admittance and radiation pattern calculations of slot antenna covered with thin plasma slab. Hodara [11] analysed the radiation characteristics deviations of slot antennas covered with a magnetized plasma sheath. Qian et al. [12] analysed the performance degradations of 


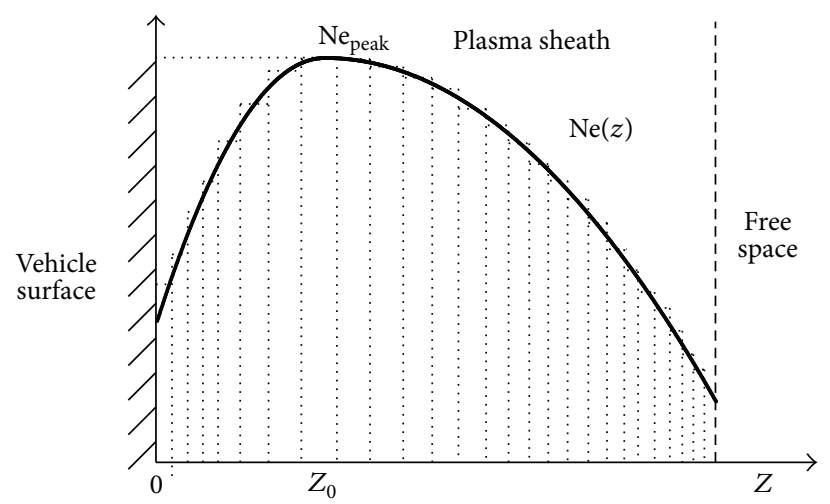

FIGURE 1: Variation profile of an electron density radially outward from vehicle surface.

C-band microstrip patch antenna covered by nonuniform plasma sheath using PLRC-FDTD algorithm. Recently, Bai [13-15] studies the admittance and radiation degradations of GPS and Beidou navigation patch antenna covered by reentry plasma sheath.

It is well known that the degradation of on-board antenna performance would lower the RF signal strength. However, by using beamforming technology, array antennas can provide a certain amount of gain to the communication link between the spacecraft and ground stations, and the received or transmitted RF signal strength would be increased. Thus, replacing the on-board single antenna with the array antennas can be adopted to mitigate the blackout problem. The process of this mitigation method is shown as follows. First, the directions of received or transmitted wave are calculated according to the navigation results and the flight attitude information of the spacecraft. Then, using beamforming technology, onboard array antenna is adjusted to point to the calculated direction. Consequently, the antenna gain and signal strength is increased, and a certain amount of signal gain is obtained to the communication link between the spacecraft and ground station.

However, the reentry plasma sheath is a reflective medium and would change the input impedance of array element, alter the mutual coupling between array elements, and degrade the beamforming performance of array antenna $[16,17]$. As a result, it is necessary to figure out this issue before this "blackout" mitigating method comes into use. Therefore, the mutual coupling property between the array elements covered by reentry plasma sheath is studied in this paper.

The remainder of this paper is organized as follows. Section 2 outlines an approximate stratified model of the nonuniform reentry plasma sheath for the EM simulation and utilizes the finite integration technique to analyse the interaction between the reentry plasma sheath and the array antenna. In Section 3, taking S-band telemetry four elements array antenna for example, self-coupling coefficient property of array element and the mutual-coupling coefficient characteristic between the adjacent and diagonal array elements covered by the reentry plasma sheath are calculated and discussed systematically. The conclusions and comments for future study are provided in Section 4.

\section{Simulation Model}

2.1. Nonuniform Plasma Sheath Stratified Modeling. In practice, the reentry plasma sheath is spatial nonuniform and variation profiles of electron density can be characterized by a bi-Gaussian function shown below [18]:

$$
\mathrm{Ne}(z)= \begin{cases}\mathrm{Ne}_{\text {peak }} \exp \left[-a_{1}\left(z-z_{0}\right)^{2}\right] & \left(0 \leq z<z_{0}\right) \\ \mathrm{Ne}_{\text {peak }} \exp \left[-a_{2}\left(z-z_{0}\right)^{2}\right] & \left(z \geq z_{0}\right) .\end{cases}
$$

Generally, the nonuniform plasma sheath can be modeled approximately by several adjacent uniform thin plasma slabs according to variation profiles of electron density. A stratified modeling method is described in [15]. The schematic diagram of the stratified model is the dotted line shown in Figure 1. Assuming that the plasma sheath is divided into $N$ layers, the complex relative permittivity of the $n$th plasma slab can be determined by

$$
\widetilde{\varepsilon}_{n}=\left[1-\frac{\omega_{p, n}^{2}}{\omega^{2}+v^{2}}-j \cdot \frac{\nu}{\omega} \cdot \frac{\omega_{p, n}^{2}}{\omega^{2}+v^{2}}\right] \cdot \varepsilon_{0},
$$

where $\omega_{p, n}$ is the plasma frequency in the $n$th layer and $v$ is the collision frequency. The electromagnetic characteristic of the nonuniform reentry plasma sheath can be established by combination of the plasma frequency $\omega_{p, n}$ and the collision frequency $v$ of each uniform plasma slab.

\subsection{Plasma-Covered Array Antenna Simulation Technique.} A square patch array antenna of four elements is designed. The array antenna is a simple 2-by-2 array, which is used to provide a representative result that reflective plasma degrades beamforming performance of array antenna in the presence of mutual coupling. The basic structure and dimensions of array element with an off-centre point feeding are shown in Figure 2(a). The layout of the array antenna on a plane is shown in Figure 2(b), and the adjacent elements are spaced at a distance of half a wavelength. The array antenna operating frequency is designed to be $2.3 \mathrm{GHz}$ which is the operating frequency of S-band TT\&C system. The relative dielectric constant and loss tangent of the substrate are 3 and 0.0013 , 


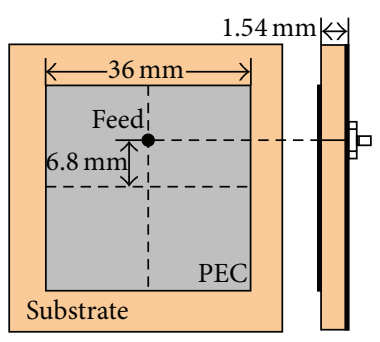

(a)

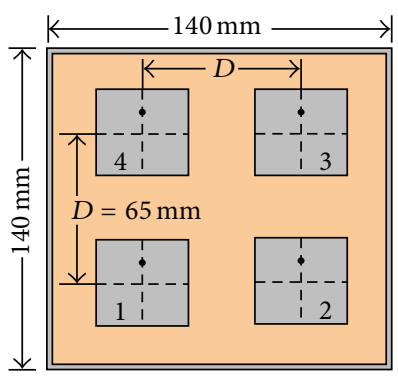

(b)

Figure 2: Basic structure and dimensions of antenna element (a) and layout of the array antenna (b).

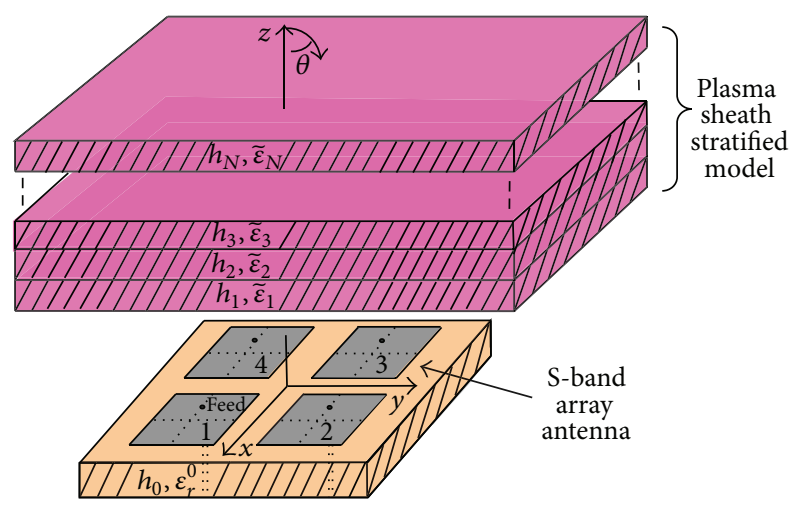

FIgURE 3: Plasma-covered array antenna simulation models.

respectively. The EM simulation model for array antenna covered with nonuniform plasma is shown in Figure 3. The interaction between the reentry plasma sheath and the array antenna is analysed using the algorithm of finite integration technique [19].

\section{Results and Discussion}

In this section, reflection coefficient property of an array element and mutual coupling characteristic between adjacent and diagonal array elements covered by reentry plasma sheath of different parameters are studied and calculated.

In the calculation, with respect to the typical reentry plasma generated in the RAM-C project [20], the bi-Gaussian plasma density distribution parameters range is confined as $\mathrm{Ne}_{\text {peak }} \sim 10^{16} / \mathrm{m}^{3} \sim 10^{18} / \mathrm{m}^{3}, \nu \sim 0.1 \mathrm{GHz} 20 \mathrm{GHz}$ and the plasma sheath thickness is set as $10 \mathrm{~cm}$. In order to simplify the multivariable problem, the growth index $a_{1}$ and the falloff index $a_{2}$ are both selected to be 0.5 , and the position of $\mathrm{Ne}_{\text {peak }}$ is fixed at the centre of the reentry plasma sheath. The simulation results are shown below.

3.1. Validity Analysis. To check the validity of this simulation method, we carry out a comparison between results using the simulation method and results using XFDTD simulation tool. In this validation, we give some typical comparison

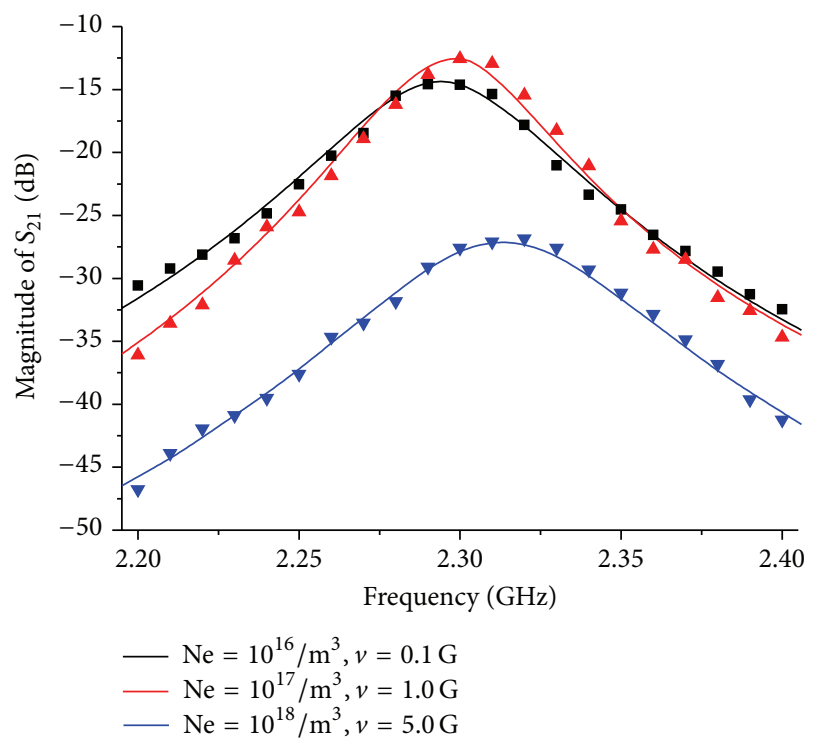

FIgURE 4: Comparisons between the results from the analysis method and the XFDTD simulation method.

results, and the plasma parameters $\mathrm{Ne}$ and $v$ are set as $10^{16} / \mathrm{m}^{3}$ and $0.1 \mathrm{GHz}, 10^{17} / \mathrm{m}^{3}$ and $1 \mathrm{GHz}$, and $10^{18} / \mathrm{m}^{3}$ and $5 \mathrm{GHz}$, respectively. The material for plasma is determined by the Debye-Drude dispersion relation model, and the layered plasma model is also applied to model the nonuniform plasma sheath In the XFDTD simulation. The $S_{21}$ parameter which is mutual coupling coefficient from the number 1 array element to the number 2 array element is presented. As shown in Figure 4, the solid lines are the results from this paper simulation method, and the scatters are the results from the XFDTD simulation. It is shown that the results from different simulation methods are coincident well with each other. Thus, the simulation method in this paper is effective and corrective.

3.2. Self-Coupling Coefficient of Array Element. Since the plasma is a reflective medium, the beamforming of array antenna would be affected by the wave reflected back into its array element. We need to calculate the self-coupling coefficient of array element. 


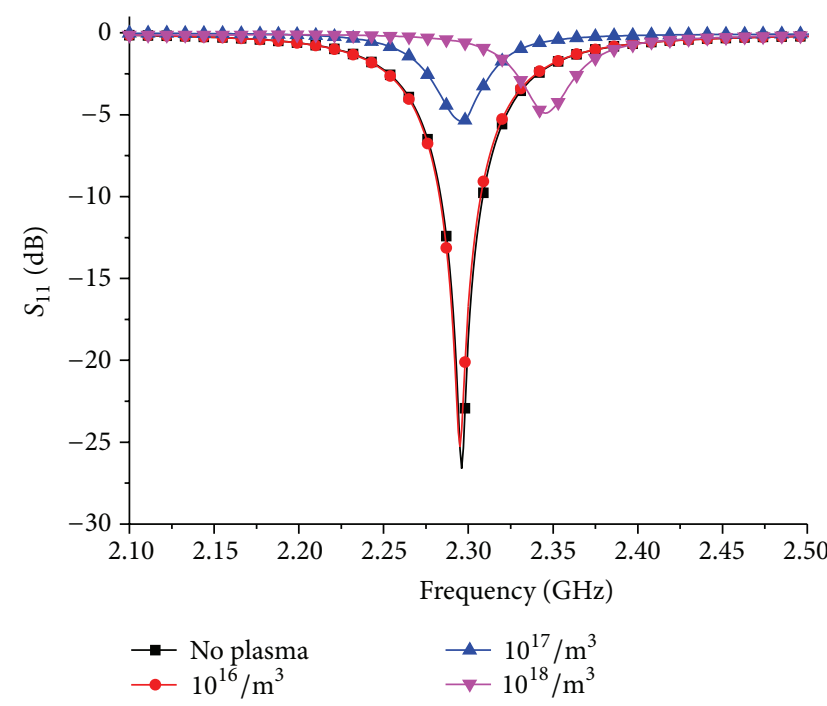

(a)

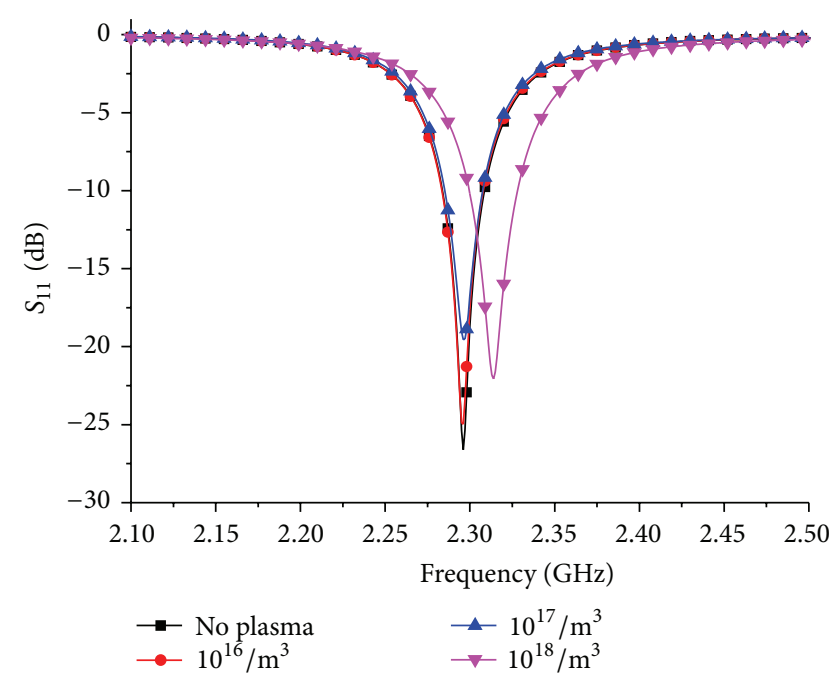

(c)

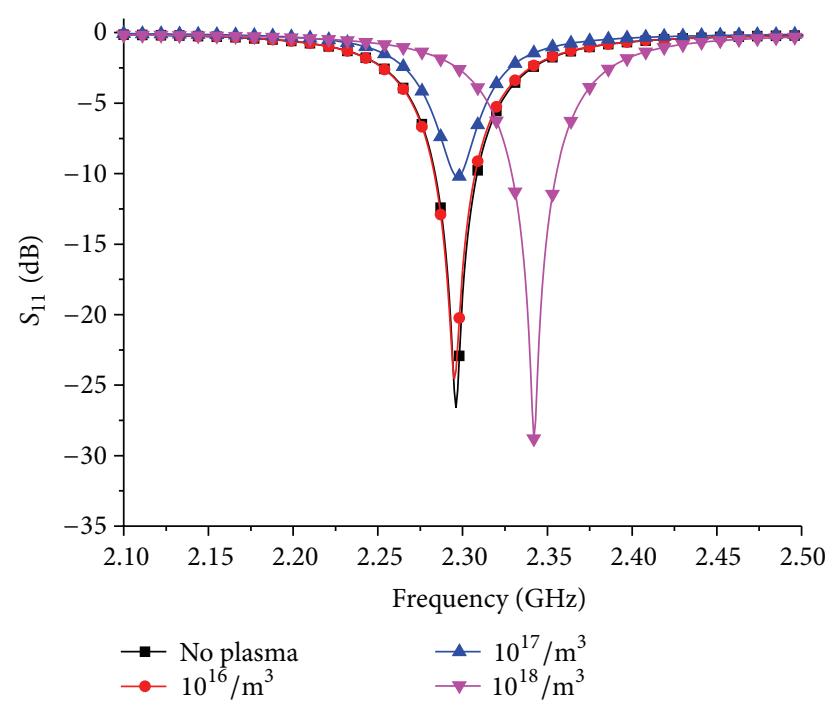

(b)

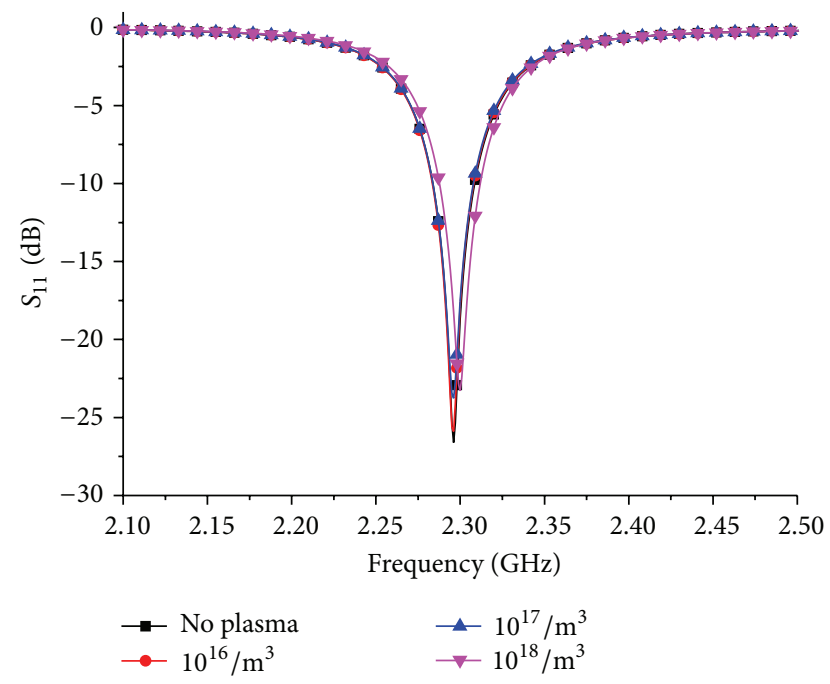

(d)

Figure 5: (a) Self-coupling coefficient $(\nu=0.1 \mathrm{GHz})$ of array element. (b) Self-coupling coefficient $(\nu=1 \mathrm{GHz})$ of array element. (c) Selfcoupling coefficient $(\nu=5 \mathrm{GHz})$ of array element. (d) Self-coupling coefficient $(\nu=20 \mathrm{GHz})$ of array element.

Because the structure of every array element is the same, the $S_{11}$ parameter which is the self-coupling coefficient of number 1 array element is presented as a demonstration. Setting $\mathrm{Ne}_{\text {peak }}$ as $0,10^{16} / \mathrm{m}^{3}, 10^{17} / \mathrm{m}^{3}$, and $10^{18} / \mathrm{m}^{3}$ for each calculation, self-coupling coefficients for collision frequencies $0.1 \mathrm{GHz}, 1 \mathrm{GHz}, 5 \mathrm{GHz}$, and $20 \mathrm{GHz}$ are shown in Figures $5(\mathrm{a}) \sim 5(\mathrm{~d})$, respectively.

The results indicate that self-coupling coefficients of the array element are deteriorating more seriously with the increase of electron density. For instance, when the collision frequency is $1 \mathrm{GHz}$, the $S_{11}$ values for $\mathrm{Ne}_{\text {peak }} 10^{16} / \mathrm{m}^{3}, 10^{17} / \mathrm{m}^{3}$, and $10^{18} / \mathrm{m}^{3}$ are $-24.5 \mathrm{~dB},-10.2 \mathrm{~dB}$, and $-2.6 \mathrm{~dB}$, respectively.

Besides, the lower the collision frequency, the more serious the deterioration of self-coupling coefficient. For instance, when the $\mathrm{Ne}_{\text {peak }}$ is $10^{17} / \mathrm{m}^{3}$, the $S_{11}$ values for the collision frequencies $0.1 \mathrm{GHz}, 1 \mathrm{GHz}$, and $5 \mathrm{GHz}$ are $-6.5 \mathrm{~dB}$, $-10.2 \mathrm{~dB}$, and $-19 \mathrm{~dB}$, respectively.

Moreover, for a large electron density (e.g., $10^{18} / \mathrm{m}^{3}$ ), the resonant frequency of the array element is adjusted to a higher frequency.

3.3. Mutual Coupling Coefficient of Adjacent Array Elements. In the former calculation, the results reveal that the plasma sheath reflects EM waves back into the array antenna. Thus, the mutual coupling characteristic of adjacent elements caused by the plasma sheath is studied in this calculation.

The $S_{21}$ parameter which is mutual coupling coefficient of the number 1 array element to the number 2 array element is presented as a demonstration for mutual coupling properties of adjacent elements. By setting plasma parameters the same 

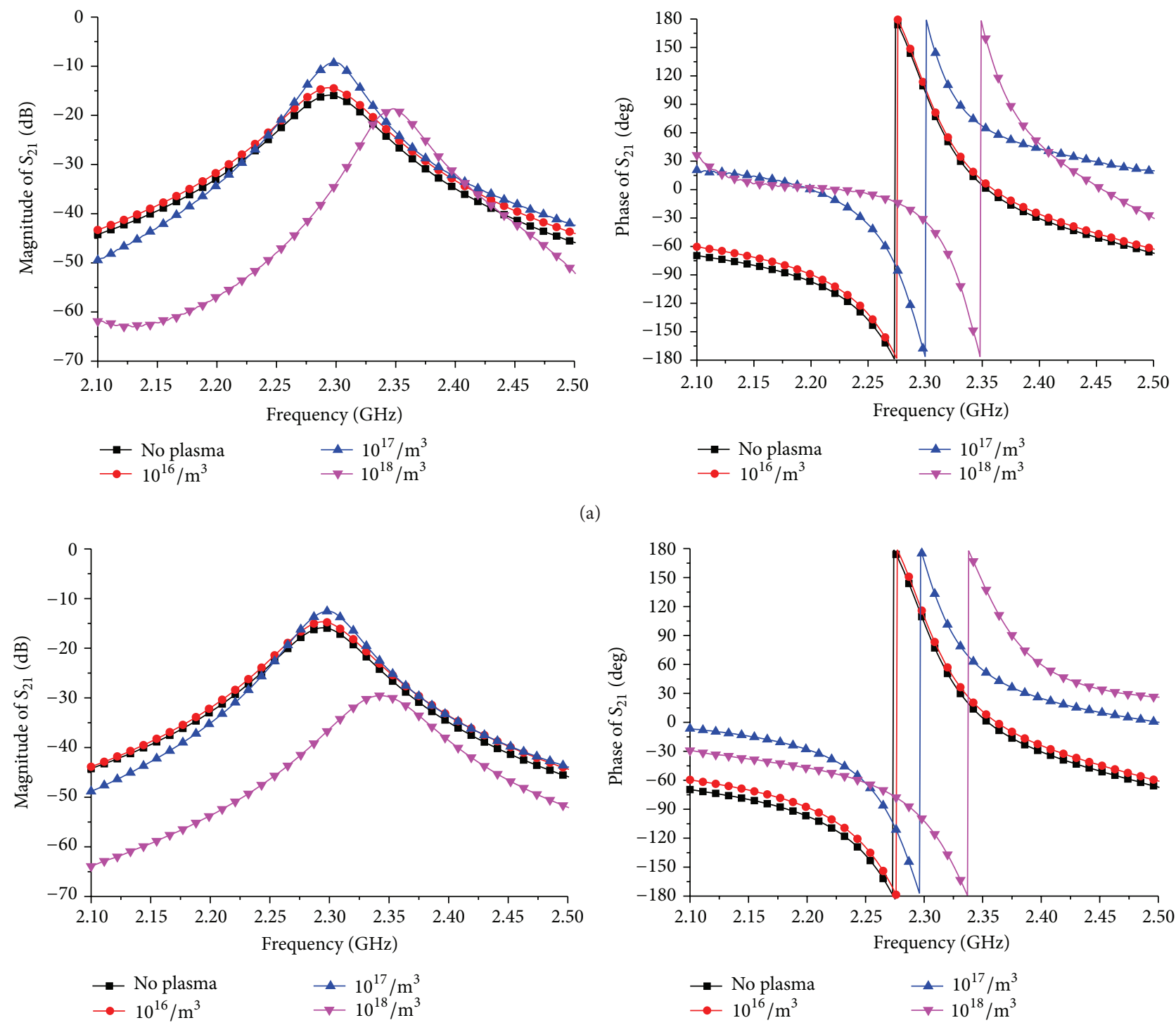

(b)
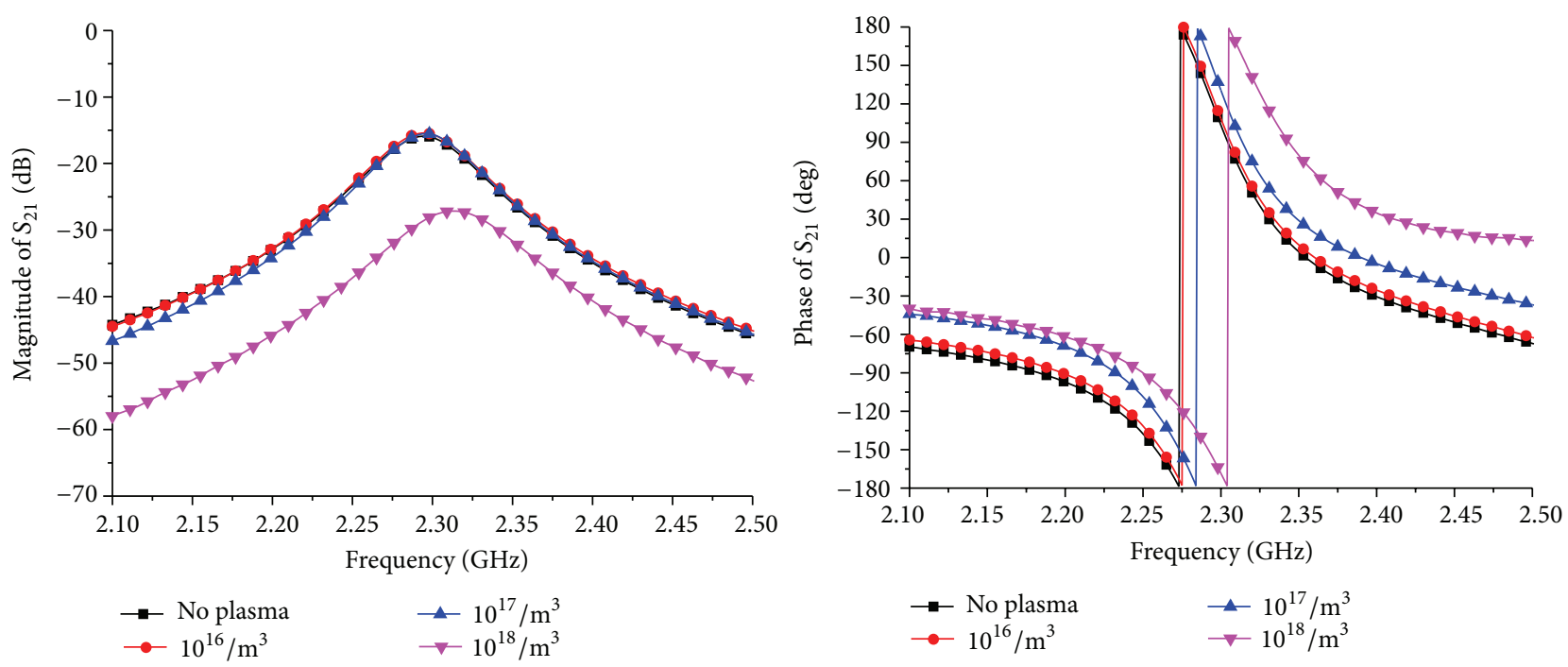

(c)

FIGURE 6: Continued. 

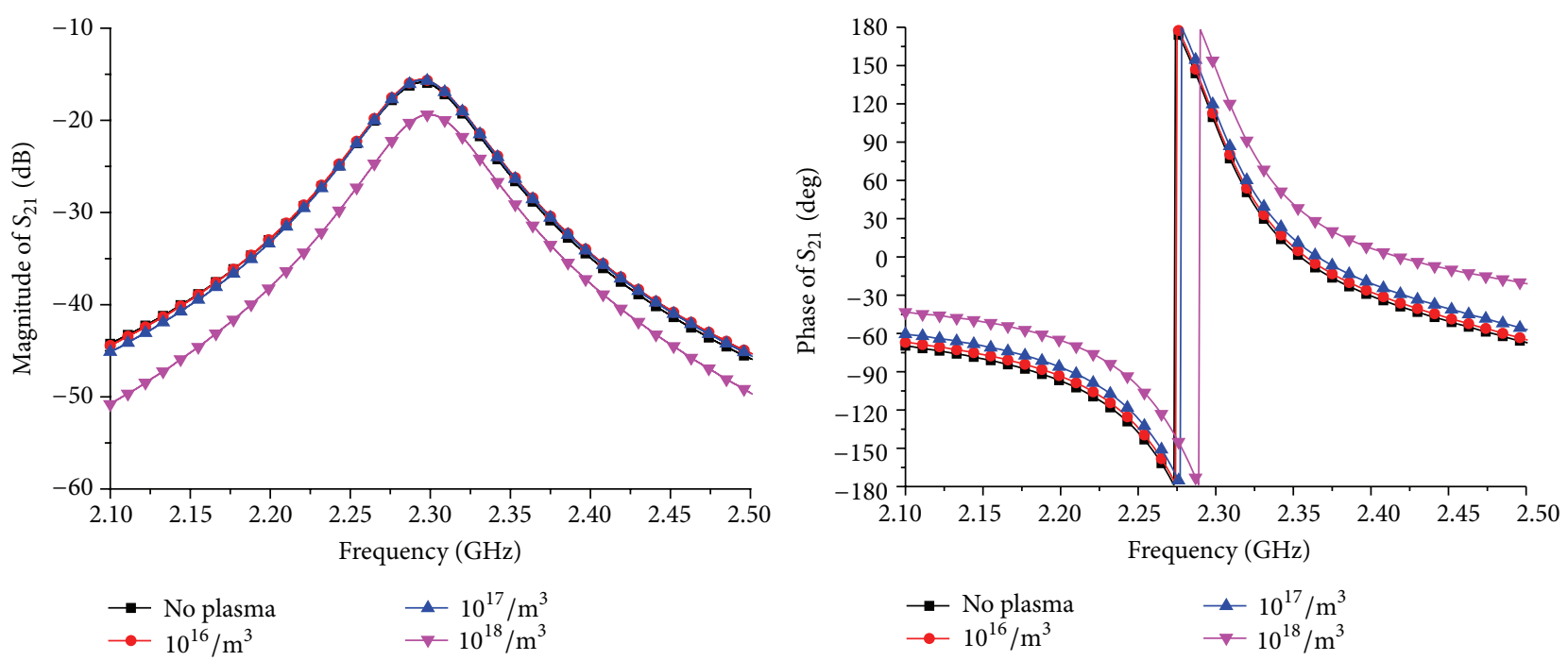

(d)

Figure 6: (a) Mutual coupling coefficient $(\nu=0.1 \mathrm{GHz})$ of adjacent elements. (b) Mutual coupling coefficient $(\nu=1 \mathrm{GHz})$ of adjacent elements. (c) Mutual coupling coefficient $(\nu=5 \mathrm{GHz}$ ) of adjacent elements. (d) Mutual coupling coefficient $(\nu=20 \mathrm{GHz})$ of adjacent elements.

as above, the mutual coupling coefficients are shown in Figures $6(\mathrm{a}) \sim 6(\mathrm{~d})$.

The results indicate that the magnitude of mutual coupling coefficients is deteriorating more seriously with the decrease of collision frequency. In particular, for the collision frequency lower than the antenna operating frequency (Figures 5(a) and 5(b)), the mutual coupling coefficients are increased when the $\mathrm{Ne}_{\text {peak }}$ are $10^{16} / \mathrm{m}^{3}$ and $10^{17} / \mathrm{m}^{3}$, whereas the mutual coupling coefficients are decreased when the $\mathrm{Ne}_{\text {peak }}$ is $10^{18} / \mathrm{m}^{3}$. For the collision frequency higher than the antenna frequency (Figures 5(c) and 5(d)), the mutual coupling coefficients change a little when the $\mathrm{Ne}_{\text {peak }}$ is $10^{16} / \mathrm{m}^{3}$ and $10^{17} / \mathrm{m}^{3}$, whereas the mutual coupling coefficients are decreased when the $\mathrm{Ne}_{\text {peak }}$ is $10^{18} / \mathrm{m}^{3}$.

The results also indicate that the phase variation relative to initial phase of mutual coupling coefficients turn bigger with the increase of $\mathrm{Ne}_{\text {peak }}$ and decrease of collision frequency.

3.4. Mutual Coupling Coefficient of Diagonal Array Element. The $S_{31}$ parameter which is mutual coupling coefficient of the number array element to the number 3 array element is presented as a demonstration for mutual coupling properties of diagonal adjacent elements. By setting plasma parameters the same as above, the mutual coupling coefficients are shown in Figures 7(a) 7(d), respectively.

The results indicate that when the $\mathrm{Ne}_{\text {peak }}$ is $10^{18} / \mathrm{m}^{3}$ and the collision frequency is $0.1 \mathrm{GHz}$, the plasma sheath deteriorates the magnitude of mutual coupling coefficients of nonadjacent elements. As for the other plasma parameters, the mutual coupling coefficients are decreased. We can conclude that the plasma sheath improves the mutual coupling property of diagonal array elements.

\section{Conclusions}

This paper presents an approximate layered EM simulation model for the nonuniform reentry plasma sheath which obeys bi-Gaussian distributions from NASA's report and utilizes the algorithm of finite integration technique to analyze the effects of the plasma sheath on the reflection coefficient property of an array element and mutual coupling property between array elements. The results indicate that reflection coefficients of an array element are deteriorating more seriously with increase of the electron density and decrease of the collision frequency. The resonant frequency of the array element is adjusted to a higher frequency by a larger electron density. The mutual coupling coefficients of array elements are deteriorating more seriously with the decrease of collision frequency. Moreover, when electron density and collision frequency are both large, plasma sheath improves the mutual coupling property of array elements, this conclusion suggests that replacing the on-board single antenna with the array antennas and using beamforming technology can be adopted to mitigate the blackout problem in this condition.

\section{Conflict of Interests}

The authors declare that there is no conflict of interests regarding the publication of this paper.

\section{Acknowledgments}

This work has been supported in part by the National Basic Research Program of China under Grant 2014CB340204, in part by the National Natural Science Foundation of China under Grant 61431010, in part by the Young Scientists Fund 


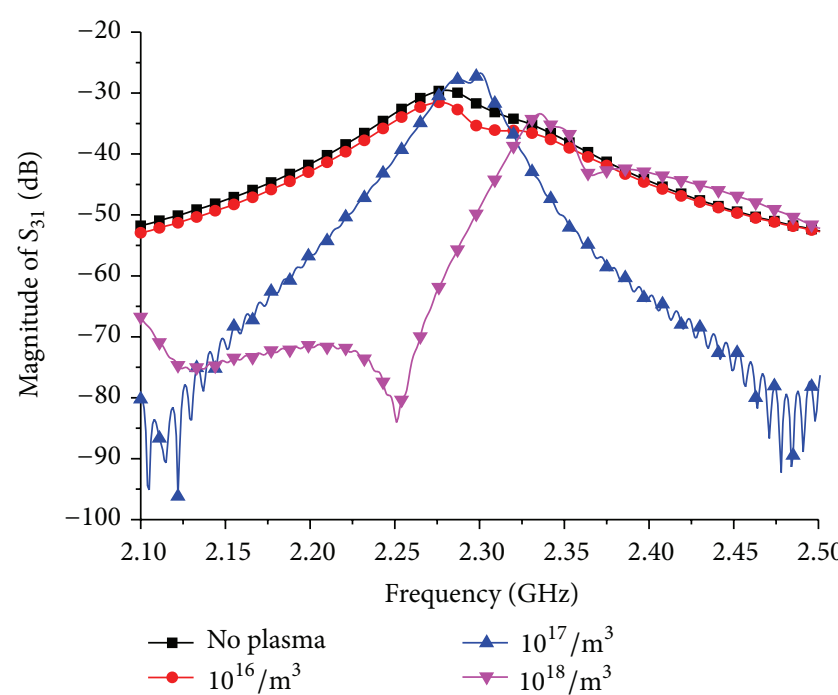

(a)

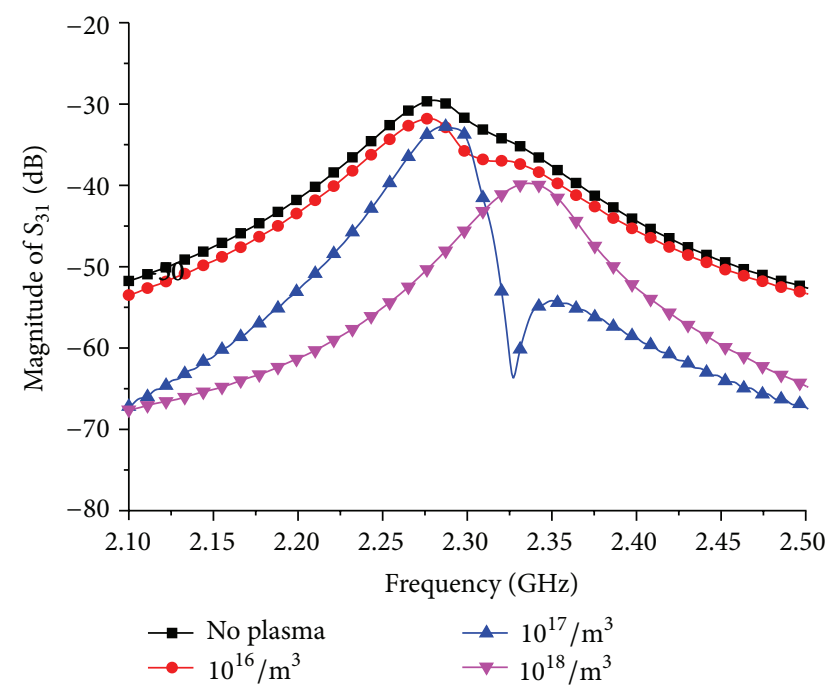

(b)

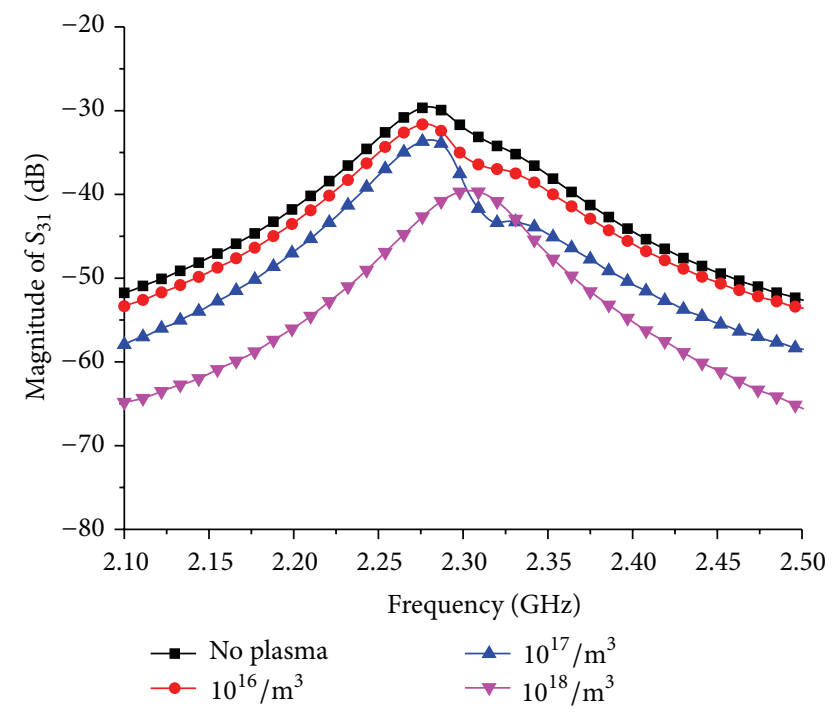

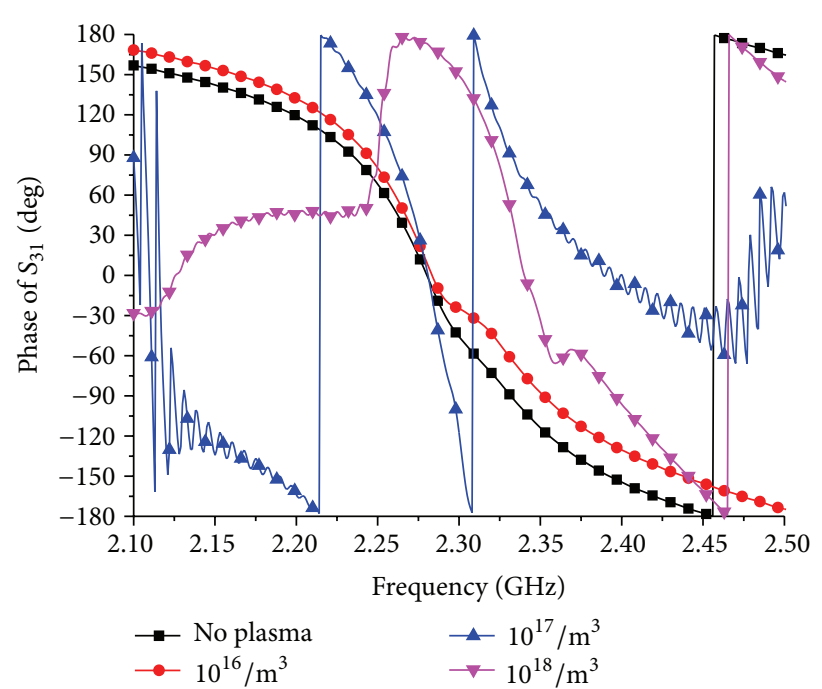
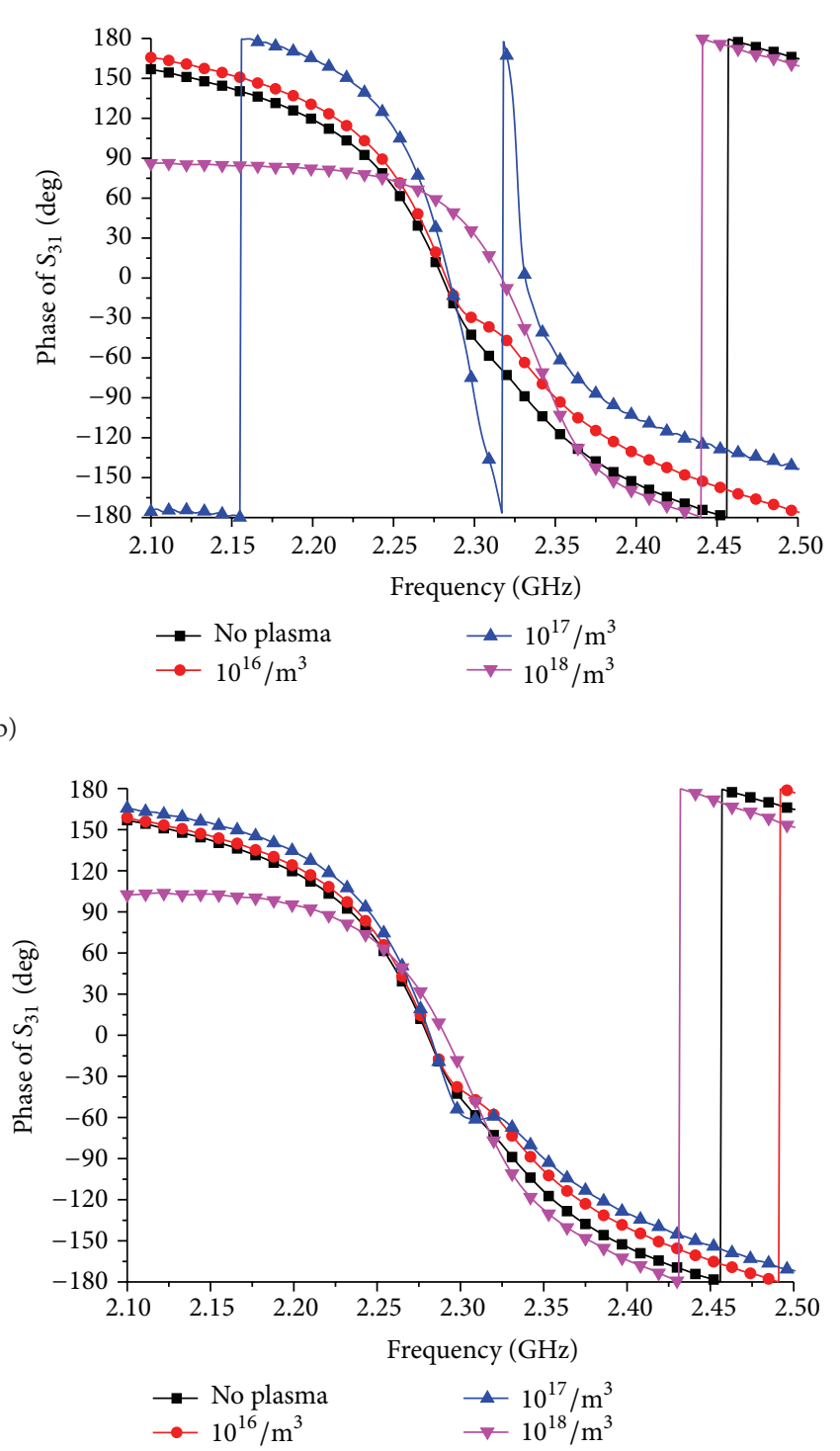

(c) 


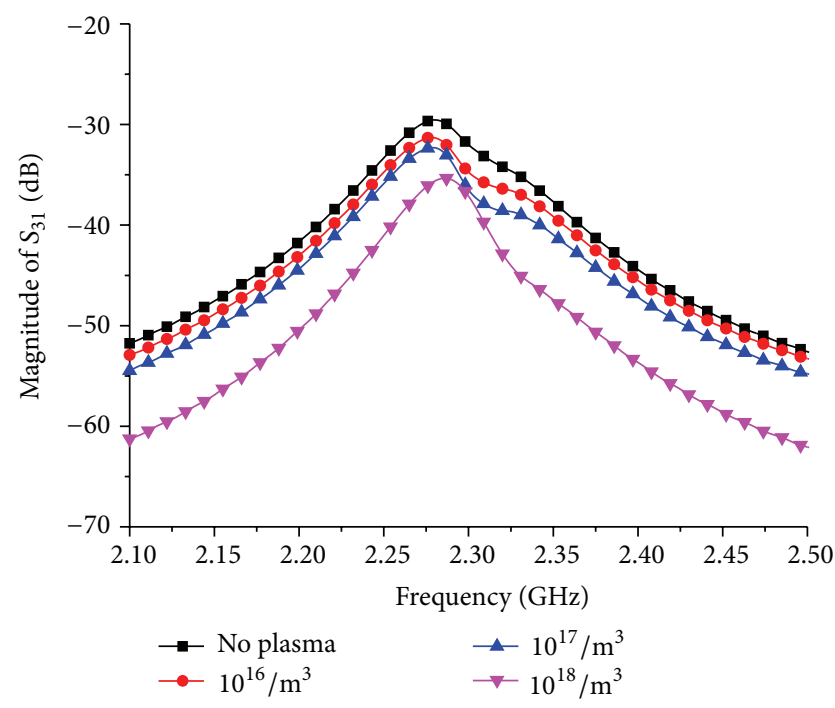

(d)

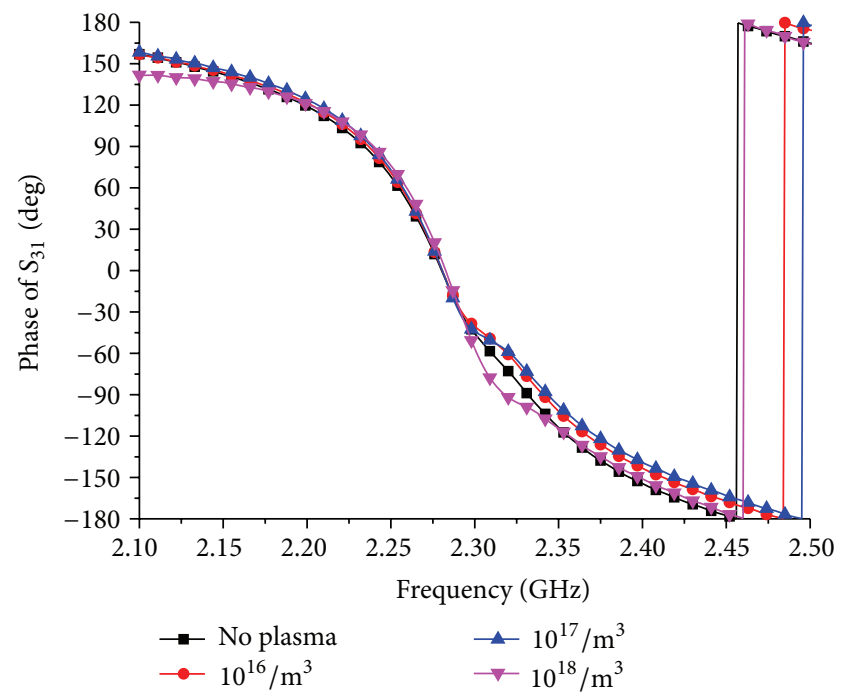

Figure 7: (a) Mutual coupling coefficient $(\nu=0.1 \mathrm{GHz})$ of diagonal elements. (b) Mutual coupling coefficient $(\nu=1 \mathrm{GHz})$ of diagonal elements. (c) Mutual coupling coefficient $(\nu=5 \mathrm{GHz})$ of diagonal elements. (d) Mutual coupling coefficient $(\nu=20 \mathrm{GHz})$ of diagonal elements.

of the National Natural Science Foundation of China under Grant 61301173, and in part by the Fundamental Research Funds for the Central Universities 72125087.

\section{References}

[1] J. P. Rybak and R. J. Churchill, "Progress in reentry communications," IEEE Transactions on Aerospace and Electronic Systems, vol. 7, no. 5, pp. 879-894, 1971.

[2] J. P. Rybak, "Causes, effects and diagnostic measurements of the reentry plasma sheath," Scientific Report AD0718428, Colorado State University, Fort Collins, Colo, USA, 1970.

[3] J. L. Poirier and W. Rotman, "Effects of the reentry plasma sheath on microwave antenna performance: Trailblazer II rocket results of 18 June 1967," Scientific Report AD865522, L. G. Hanscom Field, Bedford, Mass, USA, 1971.

[4] C. T. Swift, "Radiation patterns of a slotted-cylinder antenna in the presence of an inhomogeneous lossy plasma," IEEE Transactions on Antennas and Propagation, vol. 12, no. 6, pp. 728-738, 1964.

[5] C. T. Swift, "Admittance of a waveguide-fed aperture loaded with a dielectric plug," IEEE Transactions on Antennas and Propagation, vol. AP-17, no. 3, pp. 356-359, 1969.

[6] C. T. Swift, P. B. Gooderum, and S. L. Castellow, "Experimental investigation of a plasma covered, axially slotted cylinder antenna," IEEE Transactions on Antennas and Propagation, vol. 17, pp. 598-605, 1969.

[7] J. Galejs, "Slot antenna impedance for plasma layers," IEEE Transactions on Antennas and Propagation, vol. 12, no. 6, pp. 738-745, 1964.

[8] J. Galejs, "Admittance of a waveguide radiating into stratified plasma," IEEE Transactions on Antennas and Propagation, vol. 13, no. 1, pp. 64-70, 1965.

[9] J. Galejs and M. H. Mentzoni, "Waveguide admittance for radiation into plasma layers-theory and experiment," IEEE
Transactions on Antennas and Propagation, vol. 15, no. 3, pp. 465-470, 1967.

[10] R. Fante, "The effect of an offset impedance sheet on the admittance of a slot antenna," IEEE Transactions on Antennas and Propagation, vol. 15, no. 4, pp. 516-518, 1967.

[11] H. Hodara, "Radiation from a gyro-plasma sheathed aperature," IEEE Transactions on Antennas and Propagation, vol. 11, no. 1, pp. 2-12, 1963.

[12] Z. H. Qian, R. S. Chen, K. W. Leung, and H. W. Yang, "FDTD analysis of microstrip patch antenna covered by plasma sheath," Progress in Electromagnetics Research, vol. 52, pp. 173-183, 2005.

[13] B. Bai, X. Li, Y. Liu, L. Shi, and K. Xie, "Analysis of GPS patch antenna covered by re-entry plasma sheath," in Proceedings of the 10th International Symposium on Antennas, Propagation \& EM Theory (ISAPE '12), pp. 257-260, October 2012.

[14] L. Shi, B. W. Bai, X. P. Li, and Y. M. Liu, "Navigation antenna performance degradation caused by plasma sheath," Journal of Electromagnetic Waves and Applications, vol. 27, no. 4, pp. 518528, 2013.

[15] L. Zhao, B. W. Bai, W. M. Bao, and X. P. Li, "Effects of reentry plasma sheath on GPS patch antenna polarization property," International Journal of Antennas and Propagation, vol. 2013, Article ID 823626, 8 pages, 2013.

[16] K. E. Golden and G. E. Stewart, "Linear array and feed network performance in a reentry plasma environment," IEEE Transactions on Antennas and Propagation, vol. AP-20, no. 3, pp. 340-346, 1972.

[17] I. J. Gupta and A. A. Ksienski, "Effect of mutual coupling on the performance of adaptive arrays," IEEE Transactions on Antennas and Propagation, vol. 31, no. 5, pp. 785-791, 1983.

[18] B. Bai, X. Li, Y. Liu, J. Xu, L. Shi, and K. Xie, "Effects of reentry plasma sheath on the polarization properties of obliquely incident em waves," IEEE Transactions on Plasma Science, vol. 42, no. 10, pp. 3365-3372, 2014. 
[19] M. Clemens and T. Weiland, "Discrete electromagnetism with the finite integration technique," Progress in Electromagnetics Research, vol. 32, pp. 65-87, 2001.

[20] D. M. Dix, "Typical values of plasma parameters around a conical re-entry vehicle," Scientific Report AD295429, Aerospace Corporation, El Segundo, Calif, USA, 1962. 

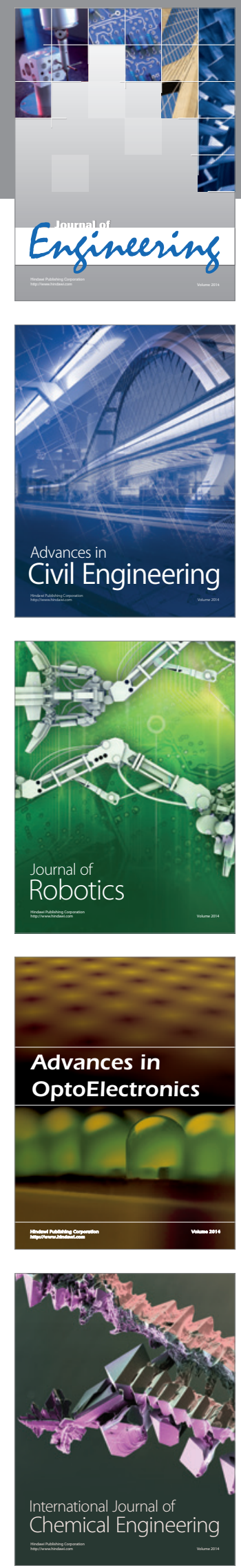

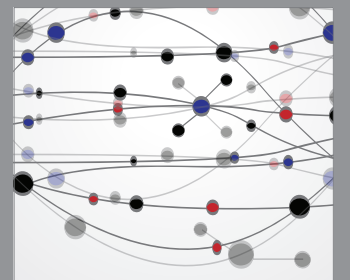

The Scientific World Journal
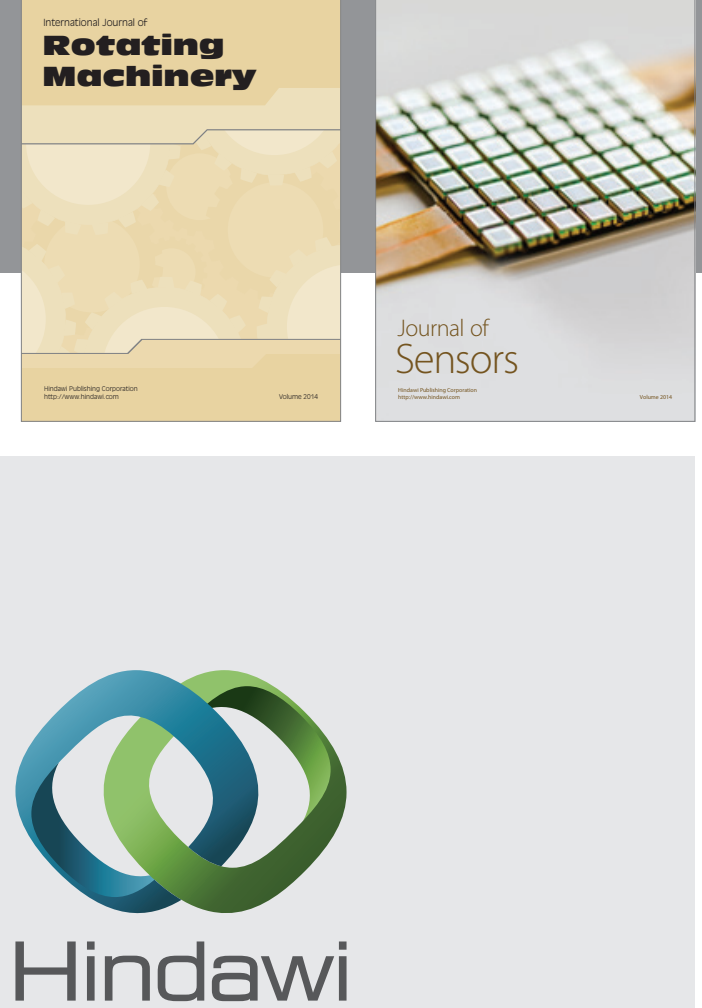

Submit your manuscripts at http://www.hindawi.com
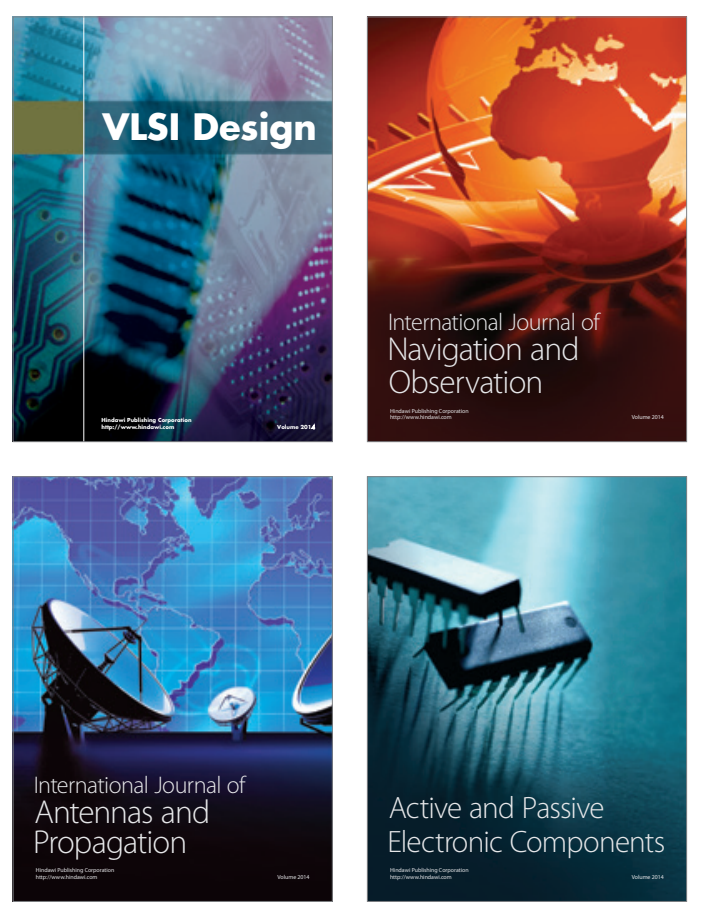
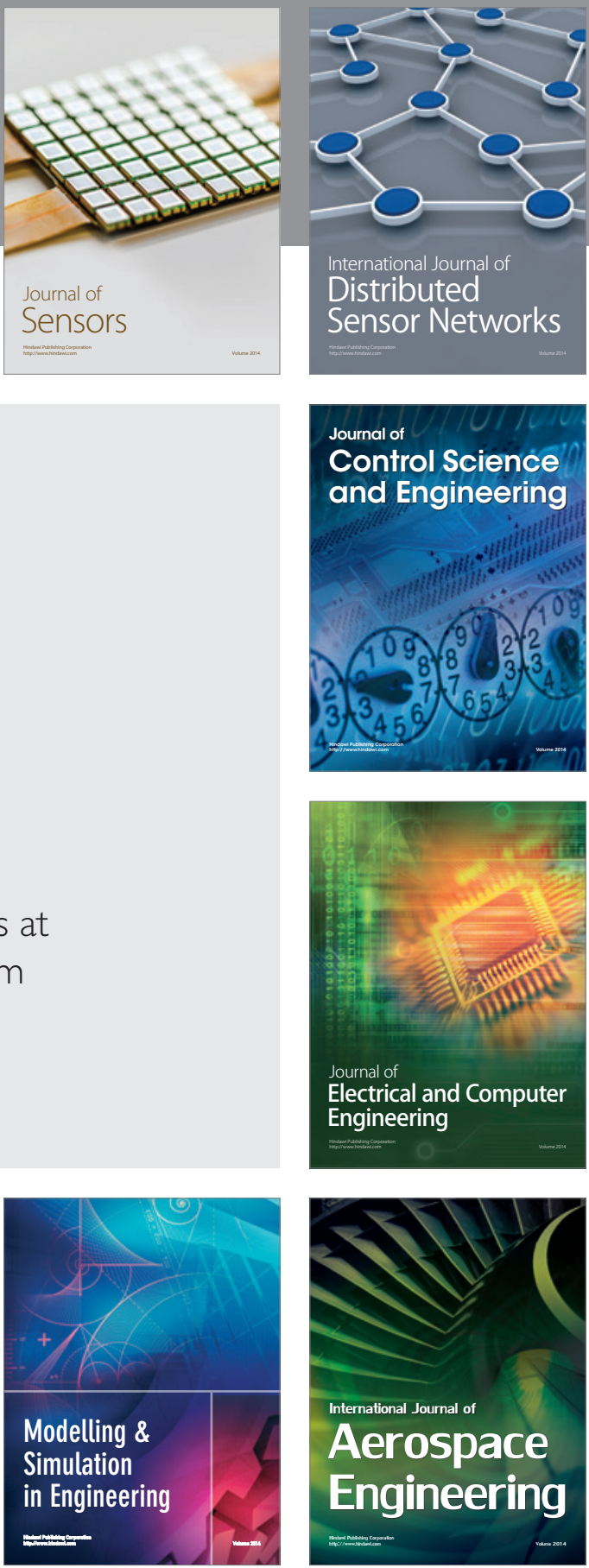

Journal of

Control Science

and Engineering
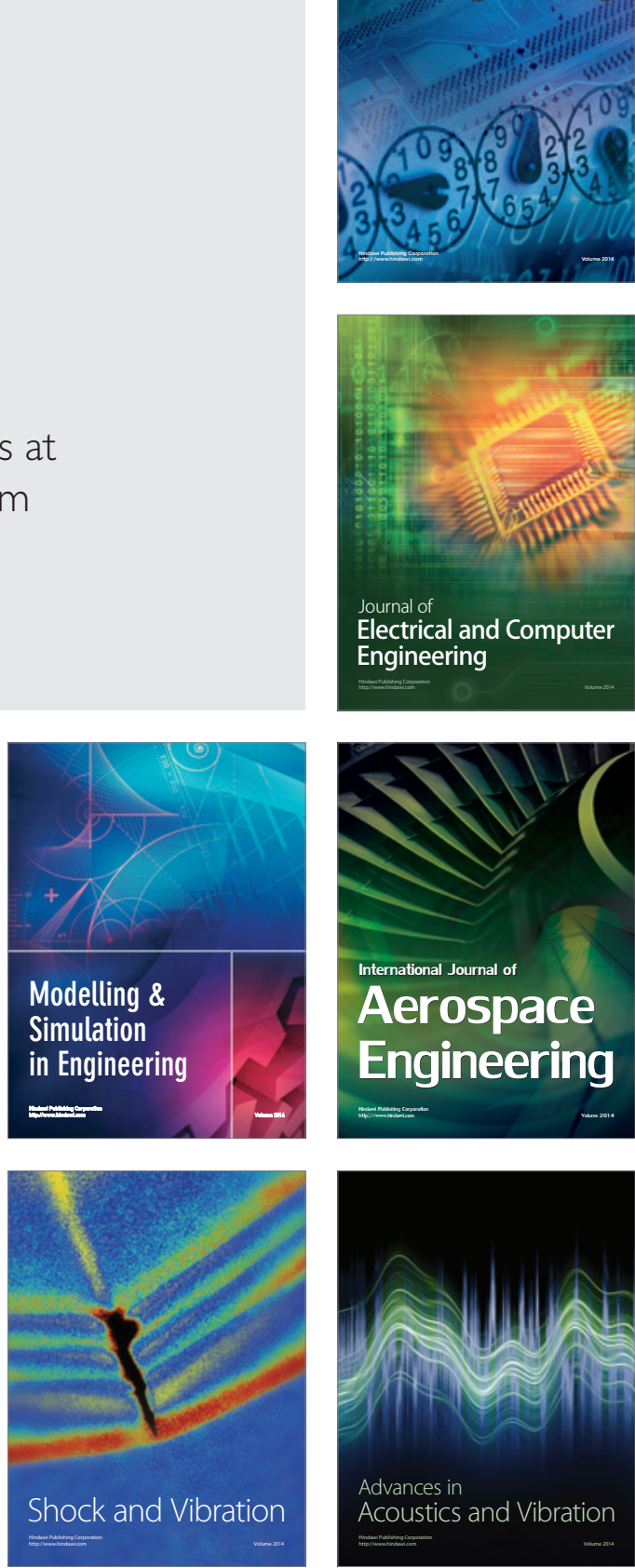